EPJ Web of Conferences 92,02115 (2015)

DOI: $10.1051 /$ epjconf/20159202115

(C) Owned by the authors, published by EDP Sciences, 2015

\title{
Investigation of Effect of Boundary Layer on Flow Structure Around a Cylinder with a Strip
}

\author{
Sedat Yayla ${ }^{1, a}$ and Süleyman Tekşin ${ }^{1}$ \\ ${ }^{1}$ Department of Mechanical Engineering, Yuzuncu Yil University, 65080 Van, Turkey
}

\begin{abstract}
In this study, the flow characteristic of the circular cylinder was placed vertically in channel which has dimensions as $8000 \mathrm{~mm}, 1000 \mathrm{~mm}, 750 \mathrm{~mm}$, lenght, width and height repectively, was investigated. The cylinder was located in boundary layer with a diameter of $60 \mathrm{~mm}$ (D) and a elastic stripwhich has a $1400 \mathrm{~N} / \mathrm{mm}^{2}$ modulus of elasticity vinyl PVC transperent film was attached behind the cylinder. Lenght of the strip (L) was 240 $\mathrm{mm} \mathrm{L} / \mathrm{D}=4$. The Reynolds number was fixed at $\mathrm{Re}=7500$. The time-averaged and instantaneous velocity vector maps, vorticity contours, Reynold shear and normal stresses, turbulent kinetic energy and frequency of shedding were obtained using the particle image velocimetry (PIV) technique. It was found that the elastic plate which exists behind the cylinder has a slight influence on the flow structure of the wake-boundary layer interaction. Values of turbulent kinetic energy, streamwise Reynold stress, transverse Reynold stresses were decreased by attaching strip.
\end{abstract}

\section{Introduction}

The flow around a cylinder is very important for researchers. Because of that lots of experiments have been done to improve flow structure.Cimbala and Garg [1] investigated the effect of the attached splitter plate on the flow characteristics downstream of a fixed cylinder and a freely rotating cylinder. The effect of fixed cylinder with an attached plate was similar to the case of a freely rotating cylinder.Zdravkovich \& Pridden emphasized the discontinuous jump in base pressure at a critical spacing of the cylinders $\mathrm{L} / \mathrm{D}=3.5$, in which $\mathrm{L}$ is the distance between centers of the cylinders and $\mathrm{D}$ is the cylinder diameter. [2] Gerrard [3]investigated the effect of length of splitter plate, attached to the circular cylinder, on the wake flow characteristics. He showed that the Strouhal number decreased when the splitter plate length was smaller than the cylinder diameter, but it increased for $1<\mathrm{L} / \mathrm{D}<2$.Kwon and Choi [4] conducted a numerical study on the control of vortex shedding in laminar flow downstream of the circular cylinder using splitter plates. They pointed out that the vortex shedding downstream of the circular cylinder completely disappears when the length of the splitter plate is longer than a critical length, which is found to be proportional to the Reynold number.Akilli and Rockwell [5] investigated the vortex formation in the junction of the flat plate andcircular cylinder in shallow water flow, using dye visualization and particle image velocimetry (PIV) techniques. Dyevisualization showed the formation of counterclockwise rotating vortices only downstream of the cylinder base, within the wake region.Kwon et al. [6] investigated the drag reduction caused by ribbons attached to circular cylinders. Theposition and length of ribbons have a significant effect on the drag reduction. Hwang et al. [7] applied a detached splitter plate for the control of flowinduced forces on a circular cylinder in their numerical study of laminar flow. They revealed that optimal location of splitter plate reduces the drag coefficient.

\section{Experimental set up and instrumentation}

Experiments were conducted on a water channel were of $8000 \mathrm{~mm} \times 1000 \mathrm{~mm} \times 750 \mathrm{~mm}$ which was made from $15 \mathrm{~mm}$ thick transparent Plexiglas sheet with upstream and downstream fiberglass reservoirs. The schematic of experimental arrangement is presented in figure 1 . The depth of the water in the test section was adjusted to 600 $\mathrm{mm}\left(h_{w}\right)$ for the present experiments. The Reynolds number was kept constant as $\mathrm{Re}=7500$. free-stream velocity of $125 \mathrm{~mm} / \mathrm{s}$. images were captured at location $250 \mathrm{~mm}\left(h_{m}\right)$ above the bottom surface of platform.

Velocity vector measurements were performed PIV system equipped by two $\mathrm{Nd}$ :Yag pulsed laser sources of a wave length of $532 \mathrm{~nm}$, each with a maximum energy output of 120 mJ.Dantec Flow Map Processor that controlled the timing of the data acquisition was used for synchronizing the camera and laser units. Also CCD camera was used to capture the images with a resolution of 1600 x 1186 pixels.350 instantaneous images were

\footnotetext{
${ }^{a}$ Corresponding author: syayla@yyu.edu.tr
} 
taken with frequency of $15 \mathrm{~Hz}$ for each capturing. In the image processing, 32 × 32 pixels with rectangular effective interrogation windows was used. During the process, an overlap of 50\% was employed. Totally 7227 $(99 \times 73)$ velocity vectors were obtained (at a rate of 15 frames per second) for an instantaneous velocity field.

The depth of water was maintained at $600 \mathrm{~mm}$ as a constant value which implies uniform flow formation in the channel owing to no variation in the flow depth (thus the average flow velocityand no fluctuation surface of the water which affects the flow structure). The Froude number, which is defined as $F r=U^{2} / g h_{w}$, based on the water depth was 0.002 that smaller than values of one.

The diameter and height of cylinder $60 \mathrm{~mm}$ and 500 $\mathrm{mm}$ respectively.Length of the elastic splitter plate were selected $240 \mathrm{~mm}$.Laser sheets were oriented parallel to the bottomsurface of the water channel at $250 \mathrm{~mm}$ height from bottom surface for images.
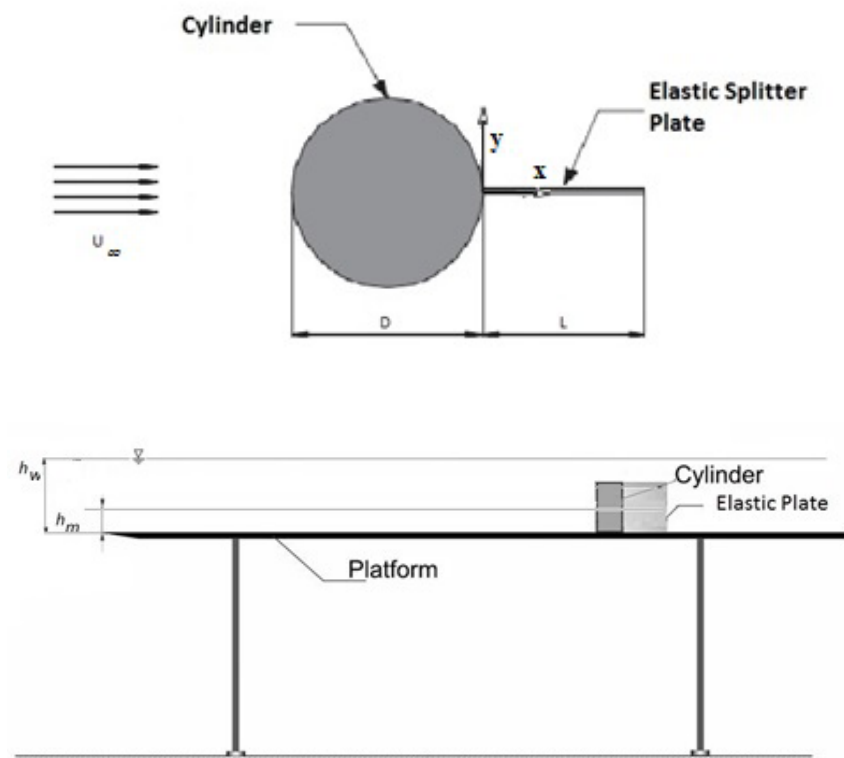

Figure 1. Schematic of the experimental system of set-up and definition of the parameters: cylinder diameter $D$, length of elastic plate $L$, laser sheet height $h_{m}$ and free-stream velocity $U_{\infty}$.

\section{Result and Discussion}

Velocity vectors $V_{\text {avg, }}$, streamwise Reynold normal stress $u^{\prime} u^{\prime} / U_{\text {avg, }}^{2}$ transverse Reynold normal stress $v^{\prime} v^{\prime} / U_{\text {avg, }}^{2}$ Reynold stresscorrelations $u^{\prime} v^{\prime} / U_{\text {avg }}^{2}$, contours of vorticity $\omega_{\text {avg, }}$, turbulent kinetik energy, are displayed infigures 2-4. Velocity vector fields, in first column, in the second column vorticity, is shown in figure 2. For vorticity contours minimum and incremental values were \pm 0.5 and 0.5 respectively. Maximum vorticity values are almost same for all cases. Foci points and saddle point which are critical location can be seen clearly for both cases. The saddle point is developed for the attached cylinder approximately $\mathrm{X} / \mathrm{D}=3.3$.

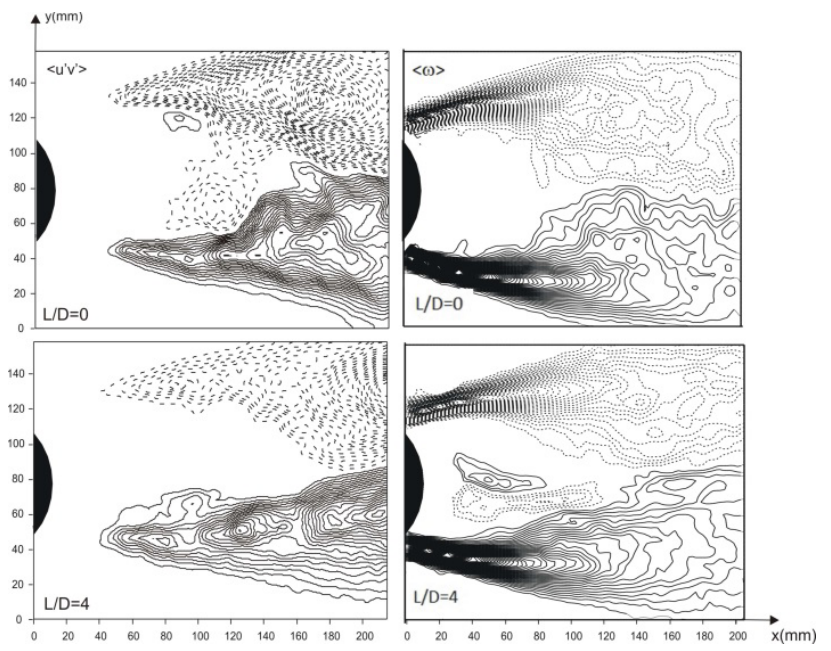

Figure 2. Time-averaged Reynold shear stress and vorticity contours $\omega_{\text {avg }}$ (minimum and incremental values arefor Reynold shear stress \pm 0.003 and 0.003 . Minimum and incremental values are $\pm 0.5 \mathrm{~s}^{-1}$ and $0.5 \mathrm{~s}^{-1}$ for vorticity respectively).

Symmetrical vortex regions can be obtained comparison bare cylinder and with elastic plate attached shape with respect to centerlines of two models.

Maximum values of streamwise Reynold normal stress $u^{\prime} u^{\prime} / U_{\text {avg, }}^{2}$, transverse Reynold normalstress $v^{\prime} v^{\prime} / U^{2}$ avg, and Reynold shear stress correlations $u^{\prime} v^{\prime} / U_{\text {avg }}^{2}$ are shown in figure 3 . While the solid lines show the positive (anticlockwise), dashed lines negative (clockwise) spanwise vorticity layers, respectively. For streamwise and transverse Reynold normal stress, minimum and incremental values are \pm 0.01 and 0.005 respectively. Reynold shear stress's minimum value is \pm 0.003 and increments are \pm 0.003 . The maximum values of the streamwise Reynold normal stresses of bare and attached body are 0.21 and 0.115 respectively while transverse Reynold normal stress was decreased from 0.2 to 0.054 .

Figure 4 showsthe contours of the normalized TKE for the two models at $\mathrm{Re}=7500$. Minimum and incremental values are \pm 0.005 and 0.01 respectively. The peak value of the TKE for the bare cylinder has a value of 0.21 and that for attached cylinder is 0.19 for $\mathrm{L} / \mathrm{D}=4$. for bare and attached body, location of maximum values approximately symmetrical with respect to cylinder centerline. As espected, peak value points were shifted far from the cylinder base. 

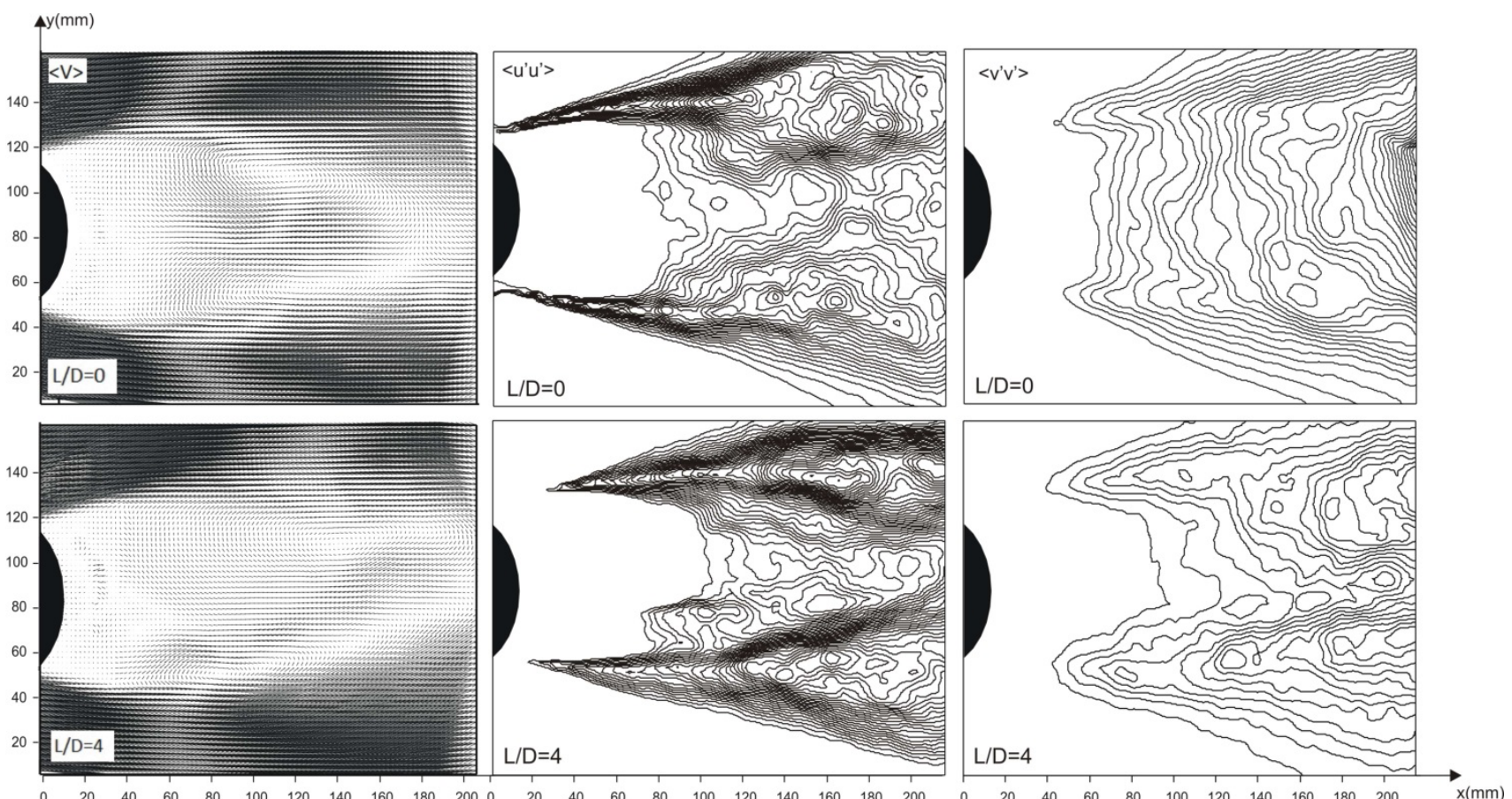

Figure 3. Time-averaged streamwise Reynold normal stress, transverse Reynold normal stress (minimum and incremental values are \pm 0.01 and 0.005 respectively).

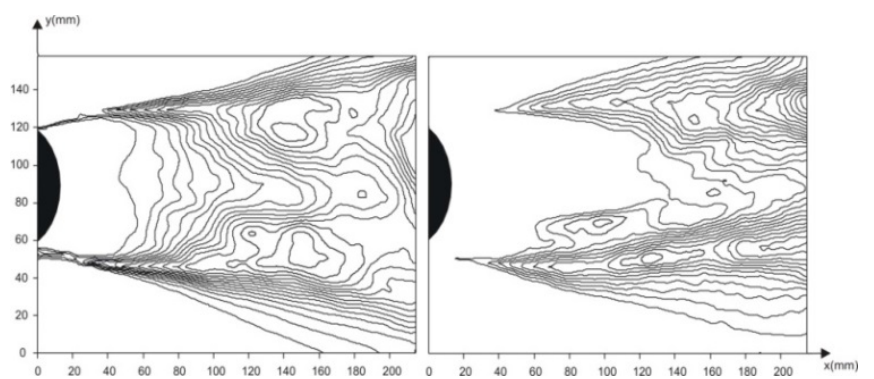

Figure 4. Turbulent kinetic energy. Minimum and incremental values are 0.005 and 0.01 respectively.

\section{Conclusion}

In this study, the passive control of the wake flow past a circular cylinder with at Reynolds number 7500 was have been investigated experimentally using the PIV technique in mechanical engineering department energy devision laboratuary at Cukurova university.An elastic plate $(\mathrm{L} / \mathrm{D}=4)$ attached behind and center location of the cylinder to control flow around the body. Laser sheet was adjusted at $250 \mathrm{~mm}$ above the bottom of the cylinder. Images were captured with angle perpendicular to the laser sheet illumination. After capturing images lots of processeswere applied to meaningful data. The maximum values of the streamwise Reynold normal stress with double peaks for the two models occur at locations of approximately $2.8 \mathrm{D}$ and $3 \mathrm{Da}$ with values of 0.21 and 0.145 respectively whereas those of transverse Reynold normal stress was decreased from 0.19 to 0.09 .

Data show us that decrase in stress correlations and turbulent kinetic energy for attached bluff body with respect to bare cylinder. In other words, splitter plate which has certain modulus of elasticity can be used to control or suppress the vorticity around the cylinder.

\section{Nomencleature}

$\begin{aligned} D & =\text { cylinder diameter } \\ D_{a} & =\text { attached cylinder } \\ L & =\text { lenght of strip } \\ R e^{\prime} & =\text { Reynolds number } \\ u & =\text { streamwise velocity component } \\ v & =\text { transverse velocity component } \\ U_{\infty} & =\text { free stream velocity } \\ <>, a v g & =\text { average } \\ u^{\prime} v^{\prime} & =\text { Reynolds shear stress correlation } \\ u^{\prime} u^{\prime} & =\text { streamwise Reynold normal stress } \\ v^{\prime} v^{\prime} & =\text { transveerse Reynold normal stress } \\ u^{\prime} & =\text { fluctuations of } u \\ v^{\prime} & =\text { fluctuations of } v \\ \omega & =\text { vorticity } \\ V & =\text { velocity vector } \\ h_{w}= & \text { height of the water } \\ h_{m} & =\text { height of laser sheet }\end{aligned}$

\section{References}

1. J.M. Cimbala, S. Garg, Flow in the wake of a freely rotatable cylinder with splitter plate, AIAA J.10011003, 29 (1991)

2. M.M. Zdravkovich, Flowinduced oscillations of two interfering circular cylinders. In Proceedings International Conference on Flow Induced 
Vibration, pp. 141-154. Bowness-on-Windermere, England, Cranfield, UK: BHRS. (1982)

3. H. Gerrard, "The Mechanics of the Formation Region of Vortices BehindBluff Bodies," J. Fluid Mech., 25, pp. 401-413. (1966)

4. K. Kwon, and Choi, H., "Control of Laminar Vortex Shedding Behind a Circular Cylinder Using Splitter Plates," Phys. Fluids, 8(2), pp. 479-486. (1996)

5. Akilli H, Rockwell D Vortex formation from a cylinder in shallow water. Phys Fluids 14:29572967 (2002)

6. S. Kwon, H., J. Cho, J.S. Park, and H.S. Choi, "The Effects of Drag Reduction by Ribbons Attached to Cylindrical Pipes," Ocean Eng., 29(15), pp. (2002)

7. J.Y. Hwang, K.S. Yang, and S.H. Sun, "Reduction of Flow-Induced Forces on Circular Cylinder Using a Detached Splitter Plate," Phys. Fluids, 15(8), pp. 2433-2436, (2003). 